\title{
Brote de triquinosis en la comarca de la Vera (Cáceres) causado por Trichinella britovi
}

\author{
J. HERRÁEZ GARCÍA, L. A. LEON GARCÍA' C. LANUSSE SENDEROS, \\ M. CORTÉS BLANCO², A. GARCÍA CABAÑAS ${ }^{3}$ \\ Servicio de Medicina Interna. ${ }^{1}$ Servicio de Urgencias, Hospital Campo Arañuelo. ${ }^{2}$ Centro \\ Nacional de Epidemiología, Instituto de Salud Carlos III. ${ }^{3}$ Servicio Central de \\ Epidemiología de la Junta de Extremadura
}

\author{
AN OUTBREAK OF TRICHINELLOSIS IN THE REGION OF LA VERA \\ (CÁCERES, SPAIN) CAUSED BY TRICHINELLA BRITOVI
}

\section{RESUMEN}

Objetivo: Descripción de los aspectos epidemiológicos y clínicos de un brote de Triquinosis (TQ) en la comarca de La Vera (provincia de Cáceres) durante los meses de enero y febrero del 2002, en relación con el consumo de carne de cerdo infectada por Trichinella britovi.

Material y métodos: Al efectuarse el primer diagnostico de sospecha de TQ se localizó la pieza cárnica infectada y todas las personas expuestas a su consumo. Se realizó encuesta epidemiológica y valoración clínica (en urgencias o consulta programada) con los siguientes exámenes complementarios: hemograma completo, urea, creatinina, iones, CPK, LDH, mioglobina y serología de Trichinella.

Resultados: Se localizaron 52 personas expuestas de las cuales 35 eran varones y 17 mujeres, con edades comprendidas entre los 2 y 86 años. El diagnóstico definitivo de TQ se estableció en 16 expuestos. Los síntomas mas frecuentes fueron: diarrea (en todos los casos), fiebre, mialgias y edema facial. La eosinofilia fue el dato analítico precoz y característico. Todos los casos diagnosticados positivizaron la serología aunque este dato no fue determinante para el inicio del tratamiento, que se comenzó ante la sospecha basada en clínica compatible y eosinofilia. No hubo complicaciones y ningún paciente requirió ingreso hospitalario. La pieza cárnica fue bloqueada lográndose interrumpir la propagación del brote.

Conclusiones: Se siguen describiendo esporádicamente brotes de TQ en España, en este caso producido por Trichinella britovi. La sospecha diagnóstica precoz y su comunicación a las autoridades sanitarias logra bloquear la carne infectada e interrumpe la propagación del brote. El tratamiento se debe indicar ante cuadro clínico compatible y la presencia de eosinofilia.

PALABRAS CLAVE: Triquinosis. Trichinella britovi. Brote.

\section{ABSTRACT}

Objective: To describe the clinical and epidemiological aspects of a trichinellosis outbreak in the region of La Vera that took place during January and Frebuary 2002, related to the consumption of pork meat infected with Trichinella britovi.

Material and methods: When the first suspected case was discovered, the presumed infected meat was checked and all the people who might have eaten it were examined. An epidemiological interview and a clinic evaluation were carried out in each patient.

Results: We found 52 exposed people that had eaten the presumptive infected meat, 35 males and 17 females, with an age ranging from 2 to 86 years old. A confirmed positive diagnosis was established in 16 patients. The most frequent symtoms were: diarrhea (present in all the cases), fever, myalgia and facial edema. Eosinophilia was an early and charac teristic analytic sign. All diagnosed cases were found to have positive serology, although this was not a required criterion to start medical tre atment. Treatmen was started once the clinical suspicion of trichinellosis was determined, based on compatible clinical signs and eosinophilia. There were no complications and none of the patients required hospitali zation.The meat sample was claimed by the Health Authorities and destroyed, thus avoiding further extension of the outbreak.

Conclusions: Sporadic outbreaks of trichinellosis have been descri bed in Spain. In the present paper an outbreak caused by Trichinella bri tovi has been studied. Early diagnosis suspicion and its communication to the Health Authorities allowed the control of the outbreak and the identification of the contaminated meat. Treatment must be started after clinical suspicion and eosinophilia.

KEY WORDS: Trichinellosis. Trichinella britovi. Outbreak.

Herráez García J, Leon García LA, Lanusse Senderos C, García Cabañas A. Brote de triquinosis en la comarca de la Vera (Cáceres) causado por Trichinella britovi. An Med Interna (Madrid) 2003; 20: 63-66.

\section{INTRODUCCIÓN}

La triquinosis (TQ) es una enfermedad parasitaria causada por el nematodo Trichinella spp., que se adquiere tras la ingesta de carne o productos cárnicos crudos o insuficientemente cocinados procedentes de animales infectados (fundamentalmente cerdo y jabalí en nuestro medio) y sin el debido control sanitario1. Existen cinco especies de Trichina descritas: T. spiralis, T. britovi, T. nativa, T. pseudoespiralis y $T$. nelsoni. Las dos primeras son las especies patógenas conocidas en España. Tras el consumo por el huésped del producto infectado la larva enquistada se libera por acción del ácido digestivo y pepsina e invade la mucosa del intestino delgado madurando rapidamente a formas adultas que aproximada-

Trabajo aceptado: 18 de octubre de 2002

Correspondencia: Jacinto Herráez García. C/ Veteranos, 3, 1A. 10300 Navalmoral de la Mata. Cáceres. e-mail: zurraquin1 @ terra.es 
mente a los siete días liberan nuevas formas larvarias que penetran en el torrente circulatorio alcanzando el músculo estriado donde se enquistan y pueden sobrevivir incluso años (1). La expresión clínica de la enfermedad viene determinada por el ciclo vital del parásito.

Describimos a continuación un brote de TQ causado por $T$. Britovi, ocurrido en la comarca de la Vera (provincia de Cáceres) en relación con la ingesta de carne de cerdo infectada.

\section{MATERIAL Y MÉTODOS}

Se procedió al estudio y seguimiento de 52 personas que asistieron a un acontecimiento familiar en Madrigal de la Vera (comarca de la Vera, Cáceres), expuestas a la ingesta de carne de cerdo infectada por T. Britovi, al efectuarse el diagnóstico de sospecha del primer caso en el Servicio de Urgencias de nuestro hospital en la tercera semana de enero del 2002. Mediante encuesta epidemiológica se determinó la pieza cárnica infectada como fuente del brote ( confirmada mediante demostración del parásito) así como el mecanismo de infestación (cuantía de la ingesta y preparación culinaria) y la posibilidad de propagación familiar o comercial. Se obtuvieron datos referentes a edad, sexo, comienzo de la sintomatología y lugar habitual de residencia. En todos los casos se efectuó valoración clínica (en urgencias o en consulta programada) y se realizaron los siguientes exámenes complementarios: hemograma completo, urea, creatinina, iones, CPK, LDH, mioglobina y serología de Trichinella. La titulación secuencial de anticuerpos IgG antiTrichinella fue realizada en el Centro Nacional de Microbiología del Instituto de Salud Carlos III de Majadahonda por inmunofluorescencia indirecta (IFI).

\section{RESULTADOS}

De las 52 personas expuestas e incluidas en el estudio y seguimiento, 35 eran varones $(67 \%)$ y 17 mujeres (33\%); procediendo $40(76 \%)$ de Madrigal de la Vera, 6 (12\%) de Villanueva de la Vera, y 6 (12\%) de El Raso y Candeleda (provincia de Ávila) (Fig. 1). Las edades comprendían entre los 2 y 86 años, con una media de edad de 42 años. Se atendieron 15 casos en Urgencias (29\%) y 37 casos en consulta programada (71\%). Se presentaron síntomas en 16 casos $(30 \%)$ estando reflejada la aparición cronológica en la figura 2. La distribución de síntomas (Fig. 3) fue la siguiente: 16 pacientes $(30 \%)$ tuvieron diarrea; 14 (28\%) fiebre; 15 (29\%) mialgias, y 8 (15\%) edema facial. En 15 pacientes (29\%) se comprobó eosinofilia (Tabla I) y en 20 (38\%) se elevaron los enzimas musculares (Fig. 3).

Se realizó la primera serología a las tres semanas de inicio de síntomas con 3 positivos, 7 dudosos y 42 negativos (título de corte 1/20). A las seis semanas se comprobaron 16 positivos $(30 \%)$ y 36 negativos (70\%) sin ningún dudoso (Fig. 4), correspondiendo las serologías positivas a los pacientes sintomáticos. Fueron tratados los 16 pacientes sintomáticos (30\%): 7 con mebendazol + prednisona (13\%) y 9 sólo con mebendazol (17\%), siendo las dosis de mebendazol: $400 \mathrm{mg}$ cada 8 horas durante 10 días, y prednisona en pauta descendente habitual también durante 10 días. En la revisión efectuada en consulta programada a las 8 semanas del inicio del brote la totalidad de pacientes afectos estaban absolutamente asintomáticos y sin complicaciones por la enfermedad, ni por el tratamiento. Nin-

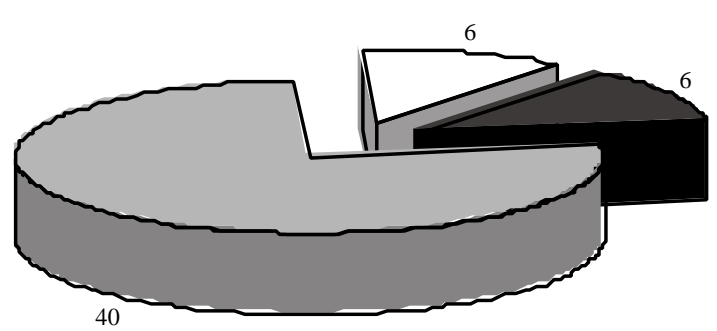

$$
\begin{aligned}
& \text { - Madrigal de la Vera } \\
& \text { El Raso-Candeleda (AV) } \\
& \text { - Villanueva de la Vera }
\end{aligned}
$$

Fig. 1. Distribución geográfica de casos expuestos.

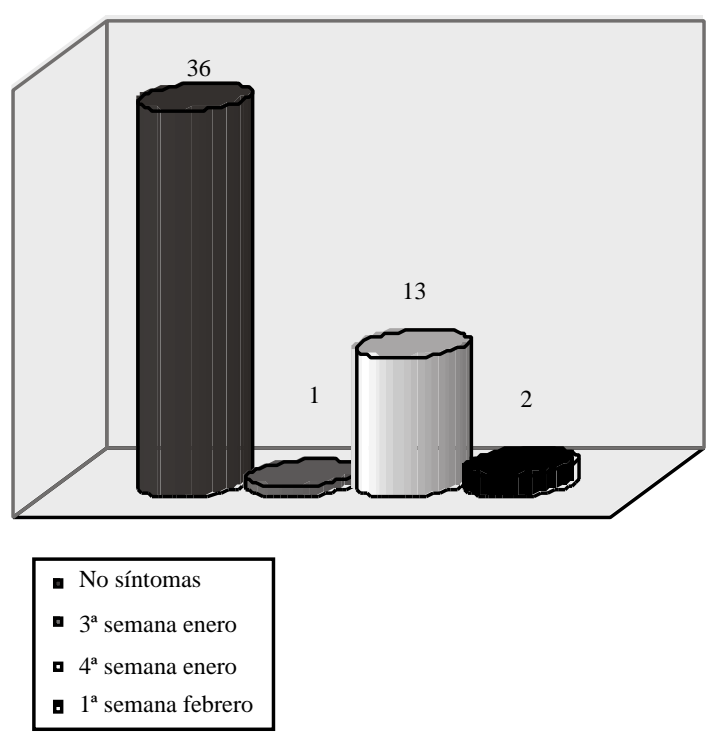

Fig. 2. Aparición cronológica de síntomas.

\section{TABLA I}

EOSINO FILIA (15 CASOS). RECUENTO TOTAL Y PORCENTUAL POR PACIENTE

\begin{tabular}{cc}
\hline Eosinófilos & Porcentaje \\
\hline 7040 & 44,8 \\
5690 & 35,6 \\
1000 & 10,9 \\
7660 & 46 \\
4130 & 42 \\
2580 & 26 \\
3170 & 28,4 \\
1570 & 11,6 \\
806 & 11,9 \\
2310 & 29,6 \\
1340 & 12,5 \\
3020 & 24,4 \\
2740 & 24,9 \\
5660 & 28 \\
750 & 11,1 \\
\hline
\end{tabular}




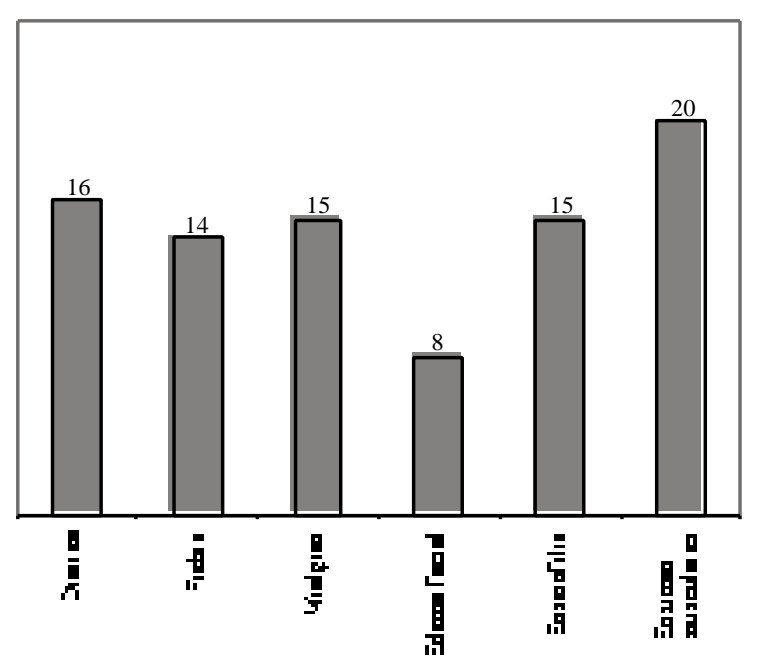

Fig. 3. Distribución de síntomas clínicos y alteraciones analíticas.

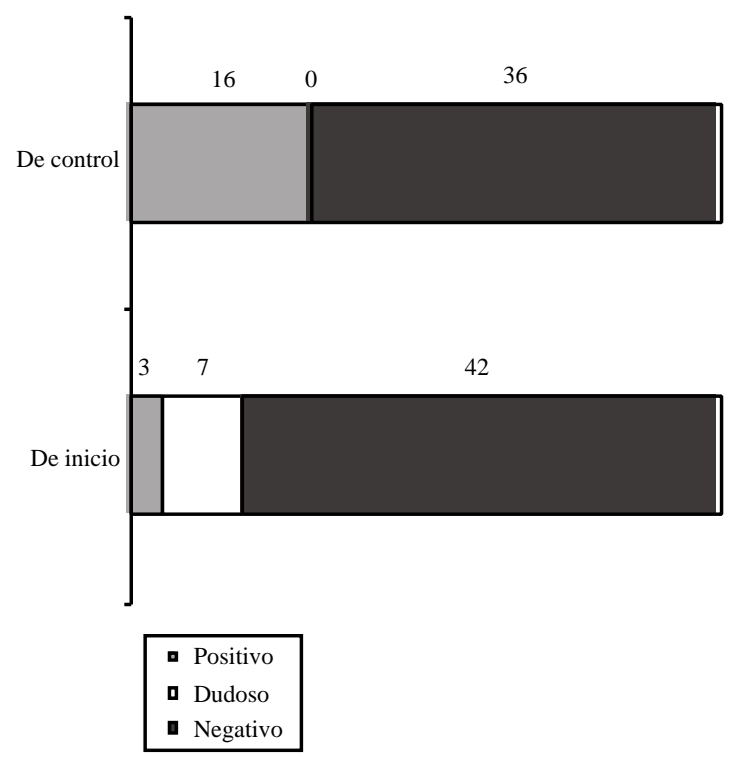

Fig. 4. Determinación secuencial de anticuerpos IgG anti-TrichineIla.

guno de los 36 expuestos restantes expresó síntomas ni signos que permitieran sospechar nuevo caso, cerrándose el seguimiento sin determinaciones analíticas posteriores.

\section{DISCUSIÓN}

La TQ humana y animal debe ser considerada en la actualidad como una enfermedad emergente y reemergente a nivel mundial, donde el aumento de su prevalencia es debido a programas veterinarios deficitarios, problemas económicos y situaciones de guerra $(2,3)$. En España no parece constituir un problema básico de salud para la población en general, aunque siguen presentándose brotes con relativa asiduidad (4-15). La mayoría de los brotes en España descritos en la literatura fueron producidos por $T$. spiralis, recogiéndose, mediante búsqueda MEDLINE desde 1989 a 2001, tres brotes producidos por T. britovi (16-18). La TQ humana presenta un periodo de incubación muy variable ( de horas hasta 50 días); y dos fase clínicas definidas: 1) enteral, en relación con la penetración en mucosa intestinal produciendo síntoma digestivos inespecíficos transitorios (nauseas, vómitos y diarrea), y 2) parenteral, en relación con la migración de la larva; produciendo mialgias, edema facial y periorbitario, conjuntivitis, fiebre, cefalea y exantema principalmente. Pueden existir complicaciones cardiológicas y en sistema nervioso central (1). La duración y severidad de los síntomas están directamente relacionadas con la cantidad de larvas ingeridas o con la viabilidad del inóculo ingerido. El estado inmunitario, la edad y la presencia de enfermedades debilitantes son factores pronósticos importantes (19). Las diferencias entre la TQ producida por $T$. spiralis y por T. britovi se analizan en el estudio de Pozio y cols. (20): en la primera los anticuerpos IgG y la CPK persisten más tiempo elevados y la sintomatología intestinal es más grave. Esto puede deberse a mayor fecundidad y tiempo de supervivencia de la larva en el músculo en el caso de $T$. spiralis. Nos sorprende el hecho de que la especia causal del brote que describimos sea $T$. britovi ya que habitualmente no se mantiene en animales domésticos (20).

El tratamiento con benzimidazoles (mebendazol, albendazol o tiabendazol) es activo en fase enteral pero inefectivo contra la larva enquistada en el músculo $(1,21,22)$. Se ha descrito fracaso terapeútico con mebendazol (22). Los corticoides se deben indicar en miositis severa y miocarditis ya sea en pauta descendente o en pulsos (23).

La posibilidad de encontrarnos ante un brote de TQ surgió ante el diagnostico de sospecha del primer paciente que acudió a urgencias por malestar general, mialgias, edema facial, fiebre y eosinofilia, con el antecedente de la ingesta dos semanas antes de carne de cerdo en un acontecimiento familiar y varias personas más presentando su misma sintomatología. Inmediatamente se comunicó a las autoridades pertinentes la posibilidad referida procediéndose a la investigación epidemiológica hasta la localización y bloqueo de la carne del cerdo infectado confirmándose la presencia de larva de Triquine lla spp. en la muestra enviada al laboratorio de la Consejería de Sanidad y Consumo de la Junta de Extremadura. En el Servicio de Parasitología del Centro Nacional de Microbiologia de Majadahonda se comprobó mediante RAPD (reacción de amplificación del ADN) que la especie de Trichina implicada era T. Britovi. Sorprendentemente los propietarios de la pieza cárnica nos comentaron que había sido sometida a control veterinario por los servicios de salud pública correspondientes de la Junta de Castilla y León, al ser remitida desde Candeleda (provincia de Ávila). Hay que asumir la posibilidad de un falso negativo aunque esta situación sobrepasa las características de este trabajo y obliga a retomar determinadas cuestiones de política sanitaria y salud pública.

En el brote que describimos la evolución cronológica de la aparición de síntomas viene determinada por la preparación culinaria de la carne: inicialmente se ingieren formas poco cocinadas (popularmente denominadas cortezas, mondongo y magro) dando lugar a los primeros casos, y con posterioridad se consume mayoritariamente en forma de embutido incrementándose el número de diagnósticos, existiendo el riesgo de propagación por consumo familiar e incluso comercial. Por tanto es absolutamente necesario localizar la fuente y en con- 
secuencia bloquear la carne infectada paralizándose la extensión del brote.

El diagnóstico definitivo de TQ se estableció en 16 pacientes. Los 36 expuestos restantes no presentaron clínica, alteraciones analíticas ni positividad serológica; presumiblemente en base a la mínima cantidad ingerida de carne infectada ya que todos reconocieron ingesta.

Las manifestaciones clínicas y la eosinofilia han sido los datos fundamentales para establecer el diagnóstico y proceder a tratamiento sin esperar a la secuencia serológica. De hecho 3 pacientes eran positivos inicialmente y los 13 restantes positivizaron el control ya estando en tratamiento. Cabe destacar la diarrea como síntoma de fase enteral presente en todos los pacientes obligando a incluir en el diagnóstico diferencial de gastroenteritis aguda la posiblidad de TQ. La eosinofilia; aunque no se demostró en un único paciente; fue el dato analítico más precoz y característico de nuestro brote, así como el descrito en la literatura (19), proporcionando una valoración fundamental a la hora de establecer dicho diagnóstico diferencial. Como dato añadido, 4 expuestos presentaron exclusivamente elevación de encimas musculares sin clínica, eosinofilia ni seroconversión; por lo que no fueron diagnosticados de infección activa y por tanto no fueron tratados. Ningún paciente requirió hospitalización, manteniéndose contacto continuo evolutivo con los propios pacientes y su respectiva atención primaria. No hemos comprobado, al menos clínicamente,

\section{Bibliografía}

1. Leo X. Liu Peter F. Weller. Helminthic Infections: Trichinella and other tissue Nematodes. In Baunwald E, Faucy AS, Kasper DL, Hauser SL, Longo DL, Jameson JL, editors. Harrison’s Principles of Internal Medicine. 15 Th ed. New York. McGraww-Hill; 2001; 1231-3.

2. Pozio E. New patterns of Trichinella infections. Vet Parasitol 2001; 98: 133-48.

3. Murrel KD. Trichinellosis: now and forevermore?. Parasite 2001; 8 (2 supl.): 11-3.

4. Sánchez Rodríguez A, Martínez López de Letona J, Arias paciencia M, Sánchez García AM, Paz Bouza J, Jarrin J, et al. Triquinosis. Estudio de 21 casos en un mismo brote. Rev Clin Esp 1982; 165: 79-84.

5. Perucha González M, Lezaun Larrumbe ME, Torres Baile JL, Campo Hernández JM, Bernal Martínez A. Brote de Triquinosis en varias localidades de la Rioja Baja. Rev Sanid Hig Pública (Madrid) 1987; 61: 1035-47.

6. De la Torre Cecilia C, Espino Aguilar R, Cárdenas Talaverón C, Canuelo Ruiz O, Garrido Palomo R, Baena Sáez J, et al. Triquinosis: presentación de 2 casos. An Esp Pediatr 1989; 30: 227-8.

7. De la Cruz de Julián I, Díaz García JM, Alvarez Lana P, García Colmenero C. Un brote de Triquinosis en Huerta del Marquesado (área básica de salud de Cañete, Cuenca). Diciembre de 1992 a Enero de 1993. Rev Sanid Hig Pública (Madrid) 1994; 68: 513-20.

8. Serrano R, Lacasa J, Velázquez J, Ziad F, Aznar R. Triquinosis: un nuevo brote epidémico causado por la ingesta de embutido de jabalí. Enferm Infecc Microbiol Clin 1989; 8: 428-31.

9. Tiberio G, Rivero M, Lanzas G, Redín D, Ardánaz E, Fernández C, et al. Triquinosis: estudio de 2 brotes en Navarra. Enferm Infecc Microbiol Clin 1997; 15: 151-3.

10. Tiberio G, Lanzas G, Galarza MI, Sánchez J, Quílez I, Martínez Arola V. Short report: an outbreack of Trichinosis in Navarra, Spain. Am J Trop Med Hyg 1995; 53: 242-2.

11. Cobo J, Gómez Cerezo J, Medrano JC, Zapico R, Cruz Martínez A, Molina F, et al. Triquinosis. Estudio de un gran brote en la Península Ibérica. An Med Interna 1991, 9: 441-4.

12. García Lechuz JM, Fernández Jáuregui C, Sánchez M, Betelu C, García Moya JB. Triquinosis en nuestra comunidad. Descripción de los últimos brotes y revisión de conjunto. FORHOS 1996; 7: 29-38. complicaciones cardiológicas de la enfermedad, su incidencia es muy baja (24), ya que no hemos efectuado sistemáticamente ECG ni ecocardiograma. Tampoco hemos recogido afectación clínica del sistema nervioso central aunque obviamente no se ha efectuado RMN a ningún paciente ante la referida ausencia de síntomas al respecto (25).

En conclusión, la infección humana por Trichinella sigue produciendo brotes esporádicos en España, debiéndose insistir en el cumplimiento de las normas sanitarias preventivas establecidas a nivel veterinario (cerdo y jabalí, fundamentalmente) y en las recomendaciones culinarias (cocinado y mantenimiento de la carne) para lograr la erradicación de la enfermedad en nuestro medio. La mayoría de brotes publicados son causados por T. Spiralis, siendo T. Britovi la especie causal del brote descrito en este trabajo. La presencia de un cuadro clínico compatible junto con eosinofilia obligan a valorar la posibilidad de TQ como diagnostico inicial y a realizar historia epidemiológica al respecto. De esta manera se logrará localizar precozmente el brote y frenar su extensión. La sospecha diagnóstica de nuestro primer caso logró, al menos, interrumpir un mayor grado de propagación familiar e incluso una posible comercialización. Las características clínicas y analíticas del brote descrito no difieren de las establecidas hasta la actualidad, mencionando la diarrea como síntoma inicial presente en todos los diagnosticados. La evolución clínica fue muy favorable, no existiendo complicaciones reseñables.

13. Muñoz JR, Ariño MD, Velilla J, Boldova JI, García Moya JB, et al. Trí quinosis. Presentación aislada de dos nuevos casos. Enferm Infecc Microbiol Clin 1985; 4: 166-9.

14. Rodríguez-Osorio M, Abad JM, De Haro T, Villa-Real R, Gómez García V. Human triquinellosis in Sourthern Spain: serologic and epidemiologic study. Am J Trop Med Hyg 1999; 61: 834-37.

15. Madrigal Díez V, Alonso Palacio J, Amo Fernández C. Brote de triquinosis en niños: seguimiento clínico durante 5 años. Bol Pediatr 1999; 39: 51-5.

16. Nerín Sánchez C, Hermida Lazcano I, Arazo Garcés P, Sardana Ferre J. Brote de triquinosis por T. Britovi. Med Clin (Barc) 1998; 11: 198-9.

17. Rodríguez E, Nieto J, Rodríguez M, Gárate T. Use of random amplified polymorphic DNA for detection of Trichinella britovi outbreaks in Spain. Clin Infect Dis 1995; 21: 1521-2.

18. López Hernández B, Gea Velázquez de Castro MT, Galicia García MD, Carlos Sabonet J. Brote epidémico por Trichinella Britovi en Granada durante la primavera del 2000. Rev Esp Salud Pública 2001; 75: 467-74.

19. Capó V, Despommier DD. Clinical Aspects on Infection with Trichinella spp. Clin Microbiol Rev 1996; 9: 47-54.

20. Pozio E, Varese P, Gómez MA, Croppo GP, Pelliccia D, Bruschi F Comparison of human trichinellosis caused by T. spiralis and by T. britovi. Am J Trop Med Hyg 1993; 48: 568-75.

21. Highlights of the Tenth International Conference on Trichinellosis August 20-24, 2000. Fontainebleau. France. Parasite 2001; 8 (2 suppl) S7-269.

22. Pozio E, Sacchini D, Sacchi L, Tamburrini A, Alberici F. Failure of mebendazol in the treatment of humans with Trichinella spiralis infection at the stage of encapsulating larvae. Clin Infect Dis 2001; 32: 638-42.

23. Vojnikovic B, Brncic N, Zamolo G, Budiselic R, Njiric S, Novak S Severe Trichinellosis cured with pulses doses of glucocorticoids. Coll Antropol 200125 Suppl: 131-5.

24. Lazarevic AM, Neskovic AN, Goronja M, Golubovic S, Bojic M, Popovic $\mathrm{AD}$. Low incidence of cardiac abnormalities in treated trichinosis: prospective study of 62 patients from a single-source outbreack. Am J Med 1999; 107: 18-23.

25. De Graef M, Smadja P, Benis J, Turpin F, Liouane M, Viaud B, et al. Neurotrichinosis: a case report with MRI evaluation. J Radiol 2000; 81: 817-9. 